\title{
Crises hipertensivas em portadores de hipertensão arterial em tratamento ambulatorial
}

\author{
HYPERTENSIVE CRISES IN BEARERS OF ARTERIAL HYPERTENSION IN AMBULATORIAL TREATMENT \\ CRISIS HIPERTENSIVAS EN PORTADORES DE HIPERTENSIÓN ARTERIAL EN \\ TRATAMIENTOAMBULATORIO
}

\author{
Nirla Gomes Guedes', Francisca Bertília Chaves Costa², Rafaella Pessoa Moreira ${ }^{3,}$ \\ Tahissa Frota Moreira ${ }^{4}$, Emília Soares Chaves $^{5}$, Thelma Leite de Araújo ${ }^{6}$
}

\section{RESUMO}

O presente estudo avaliou as características sociodemográficas e de adesão terapêutica de 27 portadores de hipertensão arterial em tratamento ambulatorial que apresentaram crises de urgências ou emergências hipertensivas e haviam sido atendidos em uma unidade de leito-dia e em uma unidade de emergência da cidade de Fortaleza-Ceará, no período de outubro de 2002 a maio de 2003. A maioria era mulher, com idade de 50 a 60 anos, pouca escolaridade, tempo de tratamento inferior a cinco anos e tempo de diagnóstico entre cinco e dez anos. O uso dos remédios foi o tratamento mais referido, seguido pela redução do consumo de sal e comparecimento às consultas. No entanto, o fato de comparecerem às consultas $\mathrm{e}$ receberem orientação parece não modificar o comportamento, uma vez que a maioria dos entrevistados não praticava exercícios físicos e demonstrava deficiência no conhecimento sobre a doença, atribuindo a elevação da pressão arterial a fatores emocionais.

\section{DESCRITORES}

Cooperação do paciente.

Enfermagem.

Hipertensão (reabilitação).

Hipertensão (enfermagem).

\section{ABSTRACT}

This study assessed the sociodemographic characteristics and the characteristics of therapeutic adhesion of 27 bearers of arterial hypertension undergoing ambulatorial treatment who had hypertensive urgencies crises or emergencies in the city of Fortaleza in the period between October of 2002 and May of 2003. The majority were women, between 50 and 60-years old, with little formal education, treatment time shorter than 5 years and time of diagnosis varying from 5 to 10 years. The use of medicine was the treatment that was most mentioned, followed by the reduction of the consumption of salt and attendance to medical appointments. However, attending the appointments and receiving orientation did not seem to change their behavior, since most of the patients that were interviewed practiced no physical exercises and demonstrated little knowledge of the illness, for they attributed the rise of the arterial pressure to emotional factors.

\section{KEY WORDS}

Patient compliance.

Nursing.

Hypertension (rehabilitation).

Hypertension (nursing).

\section{RESUMEN}

El presente estudio evaluó las características sociodemográficas y de adhesión terapéutica de 27 portadores de hipertensión arterial en tratamiento ambulatorio que presentaron crisis de urgencias o emergencias hipertensivas y habían sido atendidos en una unidad de internamiento diurno y en una unidad de emergencia de la ciudad de Fortaleza Ceará, en el período de octubre del 2002 a mayo del 2003. La mayoría fue del sexo femenino, con edad de 50 a 60 años, poca escolaridad, tiempo de tratamiento inferior a cinco años y tiempo de diagnóstico entre cinco y diez años. El uso de los remedios fue el tratamiento más referido, seguido por la reducción del consumo de sal y asistencia a las consultas. Entre tanto, el hecho de asistir a las consultas y recibir orientación parece no modificar su comportamiento, pues la mayoría de los entrevistados no praticaba ejercicios físicos y demostraba falta de conocimiento sobre la enfermedad, atribuyendo la elevación de la presión arterial a factores emocionales.

\section{DESCRIPTORES}

Cooperación del paciente. Enfermería.

Hipertensión (rehabilitación).

Hipertensión (enfermería).
1 Acadêmica de Enfermagem do $5^{\circ}$ semestre do Curso de Graduação em Enfermagem da Universidade Federal do Ceará (UFC). Bolsista PIBIC-CNPq. nirlagomes@zipmail.com.br

2 Enfermeira do Programa Saúde da Família. bertília.chaves@bol.com.br

3 Acadêmica de Enfermagem do $5^{\circ}$ semestre do Curso de Graduação em Enfermagem da UFC. Bolsista de Extensão. rafaellapessoamoreira@ hotmail.com

4 Acadêmica de Enfermagem do $5^{\circ}$ semestre do Curso de Graduação em Enfermagem da UFC. Bolsista PIBIC-CNPq. tahissa@ig.com.br

5 Enfermeira. Mestranda do Programa de PósGraduação em Enfermagem da UFC. Bolsista CAPES. emilly.e@zipmail.com.br 6 Doutora em Enfermagem. Professora Adjunta do Departamento de Enfermagem da UFC. Coordenadora do Projeto Integrado Cuidado em Saúde Cardiovascular financiado pelo CNPq. thelma@ufc.br 
Nirla Gomes Guedes Francisca Bertilia Chaves Rafaella Pessoa Moreira Tahissa Frota Cavalcante Emilia Soares Chaves Thelma Leite de Araújo

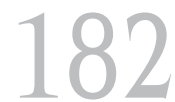

Rev Esc Enferm USP 2005; 39(2):181-8.

\section{INTRODUÇÃO}

A crise hipertensiva é resultado de elevação brusca da pressão arterial, na qual se observa níveis de pressão arterial muito elevados, acompanhados de sinais e sintomas, tais como cefaléia severa, sensação de mal-estar, ansiedade, agitação, tontura, dor no peito, tosse, falta de ar, alterações visuais e vasoespasmos ao exame de fundo de olho ${ }^{(1)}$. Pode ocorrer em indivíduos com pressão arterial habitualmente dentro de faixas de normalidade, em indivíduos portadores de hipertensão arterial ainda sem diagnóstico, como também em portadores de hipertensão arterial diagnosticada e em tratamento, sendo, nesses casos, muitas vezes em conseqüência à não-adesão ao regime terapêutico ${ }^{(2)}$.

O controle da hipertensão arterial é conseguido por meio de um programa medicamentoso, prescrito de acordo com a gravidade do quadro, e de medidas não-medicamentosas, baseadas na manutenção de um estilo de vida saudável, mediante alimentação equilibrada, prática regular de exercícios físicos, manutenção do peso corporal e abstenção do tabagismo e do etilismo. Apesar da eficácia do programa terapêutico ser bem demonstrada, observa-se com grande freqüência a não-adesão ao regime terapêutico, compreendendo como adesão o grau de cumprimento das prescrições dos profissionais que acompanham o portador de hipertensão arterial. A adesão ao regime terapêutico tem como resultados esperados o controle da pressão arterial, a redução na incidência ou retardo na ocorrência de complicações cardiovasculares e a melhoria da qualidade de $\operatorname{vida}^{(3)}$

A crise hipertensiva, decorrente da não-adesão ao tratamento da hipertensão arterial, pode representar uma situação de emergência ou urgência clínica. Na situação de emergência hipertensiva, percebem-se sinais que indicam lesões em órgãos-alvo em progressão, tais como encefalopatia hipertensiva, acidente vascular encefálico, edema agudo de pulmão, infarto de miocárdio e evidências de hipertensão maligna ou de dissecação aguda da aorta. Nesses casos, há riscos iminentes de vida ou de lesão orgânica irreversível, e os clientes devem ser hospitalizados e submetidos a tratamento com vasodilatadores de uso endovenoso ${ }^{(1)}$. Nas crises hipertensivas que constituem situação de urgência clínica, a pressão arterial pode estar bastante elevada, mas não se associam outras ocorrências, não havendo risco imediato de vida ou de dano agudo a órgãos-alvo. A correção dos níveis eleva- dos da pressão arterial deve ocorrer dentro de, no máximo, 24 horas, a permanência hospitalar é pequena, e após medicação, se a pressão demonstrar responder satisfatoriamente à terapêutica, a pessoa tem alta e é orientada a continuar seu tratamento ambulatorial. As crises hipertensivas de urgência parecem mais relacionadas a situações de estresses psicológicos associados a níveis pressóricos elevados ${ }^{(1,4)}$. A não-adesão ao tratamento da hipertensão arterial pode ocorrer devido a diversos fatores que podem estar relacionados ao paciente (hábitos de vida, crenças e hábitos culturais); à própria doença (cronicidade e ausência de sintomas); ao tratamento (efeitos incômodos das drogas); e ao acesso ao tratamento ${ }^{(5)}$.

Independente da causa da crise hipertensiva, orgânica ou emocional, os sinais e sintomas que levam o indivíduo a buscar atendimento de emergência ou urgência em serviços especializados devem ser investigados e analisados, com vistas a se saber, em especial naqueles indivíduos que estão diagnosticados e em acompanhamento por uma equipe multiprofissional, os motivos que antecedem $o$ fato.

Objetivou-se com este estudo investigar características sociodemográficas e de adesão terapêutica de portadores de hipertensão arterial que mesmo acompanhados em programas específicos, contando com prescrição e orientação de profissionais de saúde, apresentam crises hipertensivas. Ao mesmo tempo, buscou-se conhecer o que sabem sobre a doença e tratamento, suas expectativas em relação ao tratamento, os motivos que os fazem procurar serviços de urgência e emergência e a que atribuem a ocorrência das crises hipertensivas.

Mesmo que nem todas as questões suscitadas sejam respondidas com apenas uma investigação, este estudo representa uma contribuição para o conhecimento geral e para a construção de estratégias de atendimento capazes de aumentar a adesão ao tratamento, ao conseguir que os clientes apresentem as manifestações já referidas de um tratamento eficiente: níveis de pressão arterial mais próximos da normalidade, ausência de complicações cardiovasculares e melhor qualidade de vida ${ }^{(3)}$.

\section{CASUÍSTICA E MÉTODO}

O estudo teve como desenho uma abordagem exploratório-descritiva, e buscou observar, descrever e explorar aspectos de uma situação 
específica ou o conhecimento de características e problemas de um indivíduo ou grupo ${ }^{(6-7)}$.

Foi desenvolvido em dois diferentes locais: em uma unidade de referência no Estado do Ceará para o tratamento da hipertensão arterial que tem setor de urgência, denominada no estudo de Unidade A, e em uma unidade de internação que tem um setor de emergência (Unidade B). Ambos os locais são situados na cidade de Fortaleza e atendem, entre outros, pessoas em crises hipertensivas.

A população foi constituída por indivíduos portadores de hipertensão arterial de ambos os sexos, com idade superior a 18 anos e matriculados em programas de tratamento. Ademais deveriam apresentar episódios de urgência e/ou emergência hipertensiva. Em caso de urgência seriam encaminhados à Unidade de Leito-Dia e em caso de emergência, à Unidade de Internação, onde permaneciam em observação e em atendimento especializado.

$\mathrm{O}$ atendimento na Unidade de Leito-Dia da Unidade A destina-se exclusivamente aos clientes matriculados no próprio serviço e é realizado da seguinte forma: os clientes são submetidos a consultas médicas e, caso se constate quadro clínico de urgência hipertensiva, são encaminhados pelo médico para o serviço de Leito-Dia com medicação já prescrita. No local, recebem a medicação e permanecem em observação, sendo assistidos por enfermeiras e médicos que os acompanham e avaliam os sinais vitais (em especial a pressão arterial e o pulso) e o estado geral. Após uma hora de observação, a pressão arterial é medida e se os valores estiverem normais, os clientes são orientados sobre o tratamento a ser seguido e recebem alta do Leito-Dia, quando se marca nova data para a consulta de retorno que, geralmente, transcorre em um período de tempo inferior ao das consultas sem intercorrências. Caso os níveis pressóricos não tenham diminuído após a hora de observação com uso dos medicamentos, os clientes retornam ao médico para outras condutas que se façam necessárias.

A Unidade B, setor de emergência de um hospital onde parte do estudo foi desenvolvido, é ligada a um serviço público municipal da cidade de Fortaleza, destinado a clientela com alterações gerais de saúde, mas sempre em situações de emergência ou urgência. Os participantes do estudo foram identificados pelos dados da ficha de admissão e pela indicação da equipe médica e de enfermagem de plantão pela qual haviam sido atendidos em crise hipertensiva.

Para constituição da amostra, foram estabelecidos os seguintes critérios: estar em tratamento de hipertensão, como doença de base ou associada ao diabetes mellitus, coronariopatias, cardiopatia e nefropatia, esta última como doença secundária à hipertensão; ser matriculado em um programa de tratamento da hipertensão arterial e estar sendo/ter sido atendido nas Unidades A ou $\mathrm{B}$, devido a uma crise hipertensiva; estar consciente e orientado; ser capaz de se comunicar verbalmente; ter idade acima de 18 anos e aceitar participar da pesquisa de forma voluntária, após o esclarecimento dos objetivos. A amostra foi representada por onze participantes da Unidade A e dezesseis da Unidade B, no total de 27 participantes que satisfizeram aos critérios referidos.

Em respeito aos aspectos administrativos, foram encaminhados à direção das instituições ofícios de solicitação para o desenvolvimento da pesquisa, e somente após o aceite oficial iniciou-se a coleta de dados. Para atender às normas éticas da pesquisa científica, a proposta de estudo foi aprovada pelo Comitê de Ética em Pesquisa e do Complexo Hospitalar da Universidade Federal do Ceará (COMEPE) e cumpriu as recomendações da Resolução 196/96 referente a pesquisas desenvolvidas com seres humanos ${ }^{(8)}$.

A coleta de dados na Unidade A foi realizada nos meses de abril e maio de 2003 e na Unidade B de outubro de 2002 a maio de 2003, em dias e horários variados.

Em ambas as unidades, os dados foram coletados com a fonte de tipo primário, diretamente com o cliente, sob a forma de entrevista mediante preenchimento do formulário que tem como eixo as questões indicadas pelos objetivos do estudo. Não foi realizada medida da pressão arterial nos indivíduos, pois os objetivos do estudo contemplam apenas questões sociodemo-gráficas e de adesão ao tratamento. Antes de ser aplicado, o instrumento foi testado para validação das questões, com uma clientela similar, e reformulado para atender às necessidades detectadas.

Para a organização dos dados quantitativos, utilizou-se o Programa EPI_INFO 2000, sendo os resultados apresentados na forma de tabe-las. Quanto aos dados obtidos em questões abertas, estes foram descritos e comparados com a literatura.

\section{RESULTADOS E COMENTÁRIOS}

Foi incluído um total de 27 clientes portadores de hipertensão arterial, dos quais onze da Unidade A e dezesseis da Unidade B. As ca-racterísticas de sexo e faixa etária são apresentadas e comentadas a seguir.
Crises hipertensivas em portadores de hipertensão arterial em tratamento ambulatorial 
Nirla Gomes Guedes Francisca Bertilia Chaves Rafaella Pessoa Moreira Tahissa Frota Cavalcante Emilia Soares Chaves Thelma Leite de Araújo

Tabela 1 - Freqüência absoluta dos clientes atendidos nas Unidades A e B em razão da idade e sexo. (Fortaleza, 2003)

\begin{tabular}{|c|c|c|c|c|c|}
\hline \multirow[b]{2}{*}{$\begin{array}{c}\text { FAIXA } \\
\text { ETÁRIA }\end{array}$} & \multicolumn{4}{|c|}{ SEXO } & \multirow[b]{2}{*}{ Total } \\
\hline & $\begin{array}{c}\text { Masculino } \\
\text { A } \\
\mathbf{n}^{\mathbf{0}}\end{array}$ & $\begin{array}{c}\text { Feminino } \\
\text { n }^{\mathbf{o}}\end{array}$ & $\begin{array}{c}\text { Masculino } \\
\text { B } \\
\text { n }^{\circ}\end{array}$ & $\begin{array}{c}\text { Feminino } \\
\text { n }^{\circ}\end{array}$ & \\
\hline $30-40$ & 0 & 0 & 1 & 0 & 1 \\
\hline $41-50$ & 1 & 1 & 2 & 3 & 7 \\
\hline $51-60$ & 1 & 4 & 2 & 2 & 9 \\
\hline $61-70$ & 2 & 0 & 0 & 3 & 5 \\
\hline $71-80$ & 0 & 2 & 0 & 1 & 3 \\
\hline $81-90$ & 0 & 0 & 1 & 1 & 2 \\
\hline Total & 4 & 7 & 6 & 10 & 27 \\
\hline
\end{tabular}

Observa-se em ambas as unidades predomínio de pessoas do sexo feminino no total de dezessete mulheres. Estudo sobre os fatores que dificultam o controle da hipertensão arterial em 23 pacientes com história de internação por crise hipertensiva confirmam estes achados, porque encontrou que $69,9 \%$ deles eram mulheres ${ }^{(9)}$.

A presença mais significativa de mulheres com crise hipertensiva, no entanto, pode ser decorrente mais do fato de constituírem a maioria nos programas de tratamento da hipertensão arterial, do que por apresentarem menos adesão terapêutica, pois conforme outros estudos ${ }^{(3)}$, elas são mais aderentes quando comparadas com os homens. A maior presença feminina nos programas parece ser resultado de uma característica de gênero, acrescida das mudanças pelas quais têm passado as mulheres nos últimos anos, como o fato de saírem de casa para trabalhar, e acumularem funções de profissional, dona-de-casa, mãe e esposa, os quais parecem ter, comprovadamente, favorecido o au- mento da incidência das doenças cardíacas em mulheres $^{(10-11)}$

Quanto à faixa etária, percebeu-se uma concentração entre os indivíduos de 41 a 60 anos, no total de dezesseis indivíduos, chamando a atenção a presença de pessoas com idade entre $81 \mathrm{e}$ 90 anos. Segundo indicam os estudos a hipertensão arterial encontra-se diretamente relacionada à idade, e se faz mais presente a partir dos 50 anos, mas se observa aumento dos comportamentos de adesão terapêutica em indivíduos mais velhos $^{(3)}$. No estudo ora desenvolvido este fato não foi bem evidenciado. Entretanto, deve-se levar em consideração que nem sempre os idosos têm apoio familiar que lhes permita seguir adequadamente o regime terapêutico, porquanto quase sempre são dependentes para irem às consultas, seguirem o regime alimentar e mesmo para adquirirem os medicamentos e sobretudo para garantirem as tomadas nos dias e nos horários conforme prescritos.

Tabela 2 - Grau de instrução por grupo participante do estudo. (Fortaleza, 2003)

\begin{tabular}{|c|c|c|c|}
\hline \multirow{2}{*}{ NÍVEL DE INSTRUÇÃO } & \multicolumn{3}{|c|}{ UNIDADE } \\
\hline & $\begin{array}{l}A \\
n^{o}\end{array}$ & $\begin{array}{l}\text { B } \\
n^{\circ}\end{array}$ & $\begin{array}{c}\text { Total } \\
\mathbf{n}^{\mathbf{o}}\end{array}$ \\
\hline Sabiam apenas escrever o próprio nome & 2 & 4 & 6 \\
\hline Ensino Fundamental incompleto & 6 & 11 & 17 \\
\hline Ensino Médio incompleto & 1 & 1 & 2 \\
\hline Ensino Médio completo & 2 & 0 & 2 \\
\hline Total & 11 & 16 & 27 \\
\hline
\end{tabular}

Ao se observar os dados da Tabela 2, pode-se constatar que seis dos participantes sabiam apenas escrever o nome, dezessete não terminaram o Ensino Fundamental, dois tinham como nível de escolaridade o Ensino Médio incompleto e dois concluíram o Ensino Médio. O grau de instrução é um dos fatores determinantes da adesão terapêutica $^{(3)}$. A baixa escolaridade, predominante nas duas populações, deve ser considerada na escolha das estratégias para orientação e abordagem 
das consultas. Deficiências na formação escolar podem trazer dificuldade para a assimilação das orientações dispensadas pelos profissionais e influenciar na percepção da gravidade da doença, levando à aquisição de informações incompletas sobre os aspectos necessários para manter ou melhorar seu bem-estar e evitar a ocorrência de crises hipertensivas. A adesão terapêutica é influenciada pela compreensão dos portadores sobre a doença, e explica em parte os dados encontrados ${ }^{(12)}$. Na população deste estudo, porém, a baixa escolaridade é uma condição comum.

Tabela 3 - Distribuição dos entrevistados, segundo relação entre tempo de diagnóstico da hipertensão arterial e tempo de tratamento em anos. (Fortaleza, 2003)

\begin{tabular}{|c|c|c|c|c|}
\hline & \multicolumn{4}{|c|}{ INTERVALOS (ANOS) } \\
\hline & $<5$ & 5 a 10 & $>10$ & Total \\
\hline \multicolumn{5}{|c|}{ TEMPO DE DIAGNÓSTICO } \\
\hline Unidade A & 3 & 6 & 2 & 11 \\
\hline Unidade B & 6 & 5 & 5 & 16 \\
\hline Total & 9 & 11 & 7 & 27 \\
\hline \multicolumn{5}{|c|}{ TEMPO DE TRATAMENTO } \\
\hline Unidade A & 4 & 5 & 2 & 11 \\
\hline Unidade B & 8 & 5 & 3 & 16 \\
\hline Total & 12 & 10 & 5 & 27 \\
\hline
\end{tabular}

De acordo com a maioria dos participantes, tanto na Unidade A como na $\mathrm{B}$, o tratamento foi implementado tão logo se diagnosticou o quadro de hipertensão arterial. No grupo da Unidade A, três referiram ter o tempo de tratamento menor que o de diagnóstico, enquanto no grupo B, dois participantes relataram que o tratamento teve início depois de um período superior a dez anos do diagnóstico da doença e um após um período de oito anos, comportamento injustificável diante da situação.

No intuito de verificar o conhecimento dos participantes sobre sua terapêutica, solicitouse listarem espontaneamente os itens do tratamento (medicamentoso e não-medicamen-toso) prescrito no programa em que estavam matriculados.

Conforme mencionado por todos os participantes das Unidades A e B, em primeiro lugar, o tratamento prescrito para a hipertensão era constituído por remédios, aos quais atribuíam valor aparentemente maior para o controle da doença. Os achados revelaram que nove participantes da Unidade A e igual número da $\mathrm{B}$ faziam o uso de associação de medicamentos em seu esquema terapêutico. Tratamentos medicamentosos complexos, com mais de um medicamento, são reconhecidos como uma das causas de não-adesão ao tratamento(13-14). A individualização do tratamento é questão muito importante quando se objetiva simplificação do esquema terapêutico, instituição de modificações no estilo de vida, avaliação e discussão dos efeitos colaterais relacionados com o uso de medicamentos anti-hipertensivos, em conjunto com a avaliação da adesão ao tratamento proposto ${ }^{(15)}$. Para isto, é necessária uma boa relação entre os profissionais de saúde e o paciente para uma orientação personalizada e com possibilidade de adequar o tratamento aos hábitos e ao poder aquisitivo do paciente $^{(5)}$.

Ao se investigar as questões referentes ao tratamento não-medicamentoso, segundo se verificou, seu seguimento foi menos expressivo, comparativamente ao medicamentoso. De forma geral, de acordo com o referido pelos entrevistados quanto a primeiramente tomarem os medicamentos prescritos e só após serem interrogados sobre um provável tratamento não-medicamen-toso, relacionaram como prática seguida: "Não comer sal e nem gordura, caminhar três vezes por semana". "Alimentação direita, caminhar e tomar a medicação na hora certa". "Não se impressionar muito e não ser contrariada, além de não comer sal". "Fazer caminhadas e comparecer às reuniões três vezes por semana". É preciso esclarecer melhor o cliente sobre a importância do tratamento não-medicamentoso, porquanto este pode influenciar diretamente nos níveis tensio-nais, além de ser essencial para se atingir e manter uma qualidade satisfatória de vida.

Em seguida, diante da questão fechada que pedia para classificarem seu comportamento de adesão em categorias: nunca, raramente, às vezes e sempre, os participantes deram as respostas apresentadas no Quadro 1.
Crises hipertensivas em portadores de hipertensão arteria em tratamento ambulatorial 
Nirla Gomes Guedes Francisca Bertilia Chaves Rafaella Pessoa Moreira Tahissa Frota Cavalcante Emilia Soares Chaves Thelma Leite de Araújo

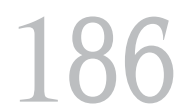

Rev Esc Enferm USP 2005; 39(2):181-8.

Quadro 1 - Distribuição dos clientes das Unidades A e B, segundo adesão ao tratamento. (Fortaleza, 2003)

\begin{tabular}{|lcccccccc|}
\hline \multirow{2}{*}{ TRATAMENTO } & \multicolumn{2}{c}{ Nunca } & Raramente & Às vezes & \multicolumn{2}{c|}{ Sempre } \\
\cline { 2 - 9 } & A & B & A & B & A & B & A & B \\
\hline Usar medicamentos & & 0 & 2 & 0 & 1 & 0 & 8 & 16 \\
Praticar exercícios físicos & 0 & 9 & 0 & 0 & 2 & 3 & 4 & 4 \\
Reduzir o consumo de sal & 5 & 1 & 1 & 1 & 2 & 0 & 8 & 14 \\
Abster-se de fumo & 0 & 1 & 0 & 1 & 0 & 1 & 5 & 13 \\
Abster-se de álcool & 0 & 0 & 0 & 1 & 2 & 2 & 3 & 13 \\
Comparecer às consultas & 0 & 0 & 0 & 1 & 0 & 2 & 11 & 13 \\
Evitar gordura e carne & 0 & 3 & 1 & 3 & 5 & 7 & 5 & 3 \\
vermelha & & & & & & & & 7 \\
Consumir frutas e verduras & 0 & 0 & 1 & 1 & 3 & 8 & 7 & 7 \\
Consumir carne branca & 0 & 1 & 2 & 1 & 3 & 6 & 6 & 8 \\
Manter peso adequado & 0 & 1 & 3 & 2 & 7 & 1 & 1 & 12 \\
Desenvolver atividades de & 0 & 6 & 4 & 2 & 1 & 4 & 6 & 4 \\
relaxamento & & & & & & & & \\
\hline
\end{tabular}

No Quadro 1 consta a situação geral de adesão ao tratamento terapêutico prescrito dos clientes entrevistados. Como se observa, os níveis de adesão ao tratamento foram divididos em quatro categorias: nunca, faz sempre, faz às vezes, faz raramente. A classificação de cada item do comportamento de adesão foi feita pelo próprio entrevistado.

Todos os clientes da Unidade A e treze da B relataram que compareciam sempre às consultas agendadas e recebiam, além da prescrição medica-mentosa, orientações sobre a necessidade de seguimento de medidas não-farmacológicas. No entanto, ao serem indagados sobre a forma de adoção de medidas não-farmacológicas, segundo se constatou, nem todos seguem o prescrito pelos profissionais de saúde, e não conseguem, assim, manter a pressão arterial em níveis controlados.

Em relação à adesão ao tratamento medicamentoso, oito participantes da Unidade A e todos da B referiram tomar corretamente a medicação prescrita e somente três clientes mencionaram não seguir de forma correta o tratamento medicamentoso, pois algumas vezes se esquecem de tomar os remédios. Para aqueles que não conseguem seguir o tratamento, seria necessário elaborar um plano terapêu-tico individualizado com vistas a facilitar o cumprimento da prescrição.

Os outros fatores considerados no Quadro 1 fazem parte do tratamento não-medicamentoso. A mudança de estilo de vida citada pela maioria dos participantes, de ambos os grupos, como sempre adotada foi a redução do consumo de sal, o maior consumo de carne branca e a abstenção do álcool e do fumo. $\mathrm{Na}$ Unidade A, apenas cinco clientes tinham histórico anterior de tabagismo ou etilismo. Tabagismo e etilismo são fatores de risco para o desenvolvimento de doenças cardiovasculares, além de dificultar o tratamento destas. A adesão ao tratamento, em relação a estes fatores, foi bastante considerável. Os alimentos gordurosos e a carne vermelha não estavam sendo sempre evitados.

Além desses itens, a maioria dos entrevistados da Unidade B referiu sempre seguir as orientações quanto à manutenção do peso adequado, às vezes consumir frutas e verduras e nunca praticar atividades de relaxamento, diferenciandose dos clientes da Unidade A, que no primeiro item tiveram como conceito prevalente às vezes, no segundo item (consumo de frutas e verduras), sempre e no terceiro item (atividades de relaxamento), sempre.

A prática de exercícios físicos foi a conduta terapêutica menos observada nos dois grupos. Há evidências de que a prática regular de exercícios físicos produz redução das pressões sistólica e diastólica, e controla os desvios metabólicos fre-qüentemente associados no cliente portador de hipertensão. Ademais, outro aspecto ressaltado como benefício de um programa regular de exercícios seria o de aliviar a tensão emocional. Este fato poderia justificar a ligação observada nos entrevistados entre a falta de adesão à prática de exercícios físicos e as atividades de relaxamento. A maior parte das crises hipertensivas é oriunda de uma situação de aumento do nível do estresse e a correção dessa situação poderia regularizar a pressão $\operatorname{arterial}^{(4)}$.

Em relação aos conhecimentos referidos pelos clientes sobre hipertensão arterial, confor- 
me se observou nos entrevistados da Unidade A, diziam respeito aos sinais e sintomas (tontura, dor de cabeça, mal-estar), às possíveis conseqüências da doença (acidente vascular cerebral (AVC) e trombose), e aos fatores de risco para o desenvolvimento da hipertensão (estresse, dieta e medicação). Na clientela da Unidade B, o conhecimento foi relacionado às possíveis conseqüências da doença e à importância de seu tratamento. No entanto, nenhum cliente da Unidade A apresentou como expectativa para o tratamento a cura da doença, mas todos esperavam "manter a pressão em níveis normais para viver bem".

Cabe aos profissionais de saúde esclarecer não só o tratamento, mas, de acordo com o nível instrucional de cada paciente, o significado da doença e suas implicações fisiopatológicas. Desse modo, os profissionais de saúde precisam ser, antes de tudo, educadores, facilitadores das transformações nas mentes humanas. Para isso, é necessário haver uma boa comunicação/relação entre o profissional e o cliente, e a valorização deste como agente do próprio cuidado.

Ao responderem sobre o possível motivo da ocorrência da crise hipertensiva que os fez procurar o serviço de urgência, os participantes da Unidade A citaram: estresse, raiva, nervosismo, problemas e preocupações com a família, como fatores desencadeantes do descontrole e oscilações da pressão arterial. Entretanto, dois clientes atribuíram a crise hipertensiva à falta de medicação, e dois não a associaram a nenhum motivo específico, pois, segundo eles, não houve nada de anormal que os levasse a ter essa alteração clínica e nem mesmo sabiam no momento da consulta que estavam com valores tão elevados. Isto confirma a pouca sintomatologia associada ao quadro. A procura pelo serviço de emergência dos participantes da Unidade B deu-se, em todas as situações, mediante o aparecimento de sinais e sintomas considerados pelos clientes como decorrentes da elevação da pressão arterial: mal-estar, tonturas, escotomas e cefaléias.

De modo geral, entretanto os participantes relataram o fator emocional como o maior responsável pela elevação da pressão arterial. No cotidiano destas pessoas, tais alterações emocionais são comuns e muitas vezes não podem ser evitadas. E se não são encaradas com calma e tranqüilidade, mesmo que sigam o tratamento medicamentoso e tenham hábitos saudáveis de estilo de vida, a pressão arterial eleva-se. Portanto, devem esses clientes participar de atividades grupais e de relaxamento, com o objetivo de aprenderem a conviver com os problemas, e, assim, evitar conseqüências, como a elevação dos níveis de pressão arterial.
Indagados sobre o número e tipos de ocorrências para a busca por um serviço de urgência e emergência nos últimos doze meses, quatro entrevistados da Unidade A e doze da Unidade B confirmaram a procura.

Ao tomar conhecimento de que o cliente ora acompanhando em um programa de tratamento vem apresentando crises hipertensivas, e necessita de atendimento especializado, os profissionais precisam buscar estratégias mais eficazes para, junto com o cliente e familiares, encontrar formas de evitar essas complicações. Muitas vezes o serviço de origem desconhece a procura à urgência e à emergência, ou se sabe, não se preocupa em investigar os porquês envolvidos na situação.

\section{CONCLUSÃO}

Apesar de estudos demonstrarem ser relativamente pequena a incidência de crises hipertensivas, em torno de $1 \%{ }^{(16)}$, nesta experiência no atendimento a portadores de hipertensão, ela é referida com mais freqüência, e até leva as pessoas, acompanhadas em programas de tratamento da hipertensão arterial, a buscarem atendimen-to clínico por apresentarem episódios de crises hipertensivas. Diante desses aspectos, desenvolveu-se esse estudo para se avaliar essas pessoas e detectar as possíveis causas que as levaram a terem intercorrências e a buscarem unidades de urgência e emergência.

O estudo incluiu, no total, 27 clientes que satisfizeram aos critérios de estarem em tratamento em programas específicos e terem sido atendidos em serviços de atendimento especializado, apresentando níveis bastante elevados da pressão arterial, característica de crise hipertensiva.

Conclui-se, com base na análise dos dados obtidos, que a adesão ao tratamento da hipertensão, no grupo avaliado, não está sendo suficiente para se evitar problemas decorrentes da doença e pode justificar a ocorrência do quadro clínico de crise hipertensiva. Segundo observou-se a nãoadesão ao tratamento pode decorrer, principalmente, da falta de esclarecimento dos clientes pela equipe de saúde, da dimensão real do problema da hipertensão e da necessidade de manutenção adequada e permanente dos cuidados gerais e cumprimento do tratamento medicamen-toso e nãomedicamentoso. Neste contexto, evidencia-se a importância da atuação do profissional de saúde no processo educativo do cliente, na facilitação de conhecimento de sua doença e do tratamento respectivo, com vistas ao aumento dos índices de adesão ao tratamento.
Crises hipertensivas em portadores de hipertensão arterial em tratamento ambulatorial 
Nirla Gomes Guedes Francisca Bertilia Chaves Rafaella Pessoa Moreira Tahissa Frota Cavalcante Emilia Soares Chaves Thelma Leite de Araújo
Correspondência: Nirla Gomes Guedes Rua Dr. Alfredo Weyne, 55 - Ap. 1002 - BI. B B. de Fátima - Fortaleza CEP -60415-520 - CE
Espera-se com este estudo contribuir de alguma maneira para que os profissionais de saúde que lidam com pessoas portadoras de hipertensão arterial consigam esclarecê-las devidamente sobre sua doença e tratamento, no intuito de diminuir o índice de alterações clínicas conseqüentes da hipertensão arterial. Reconhece-se a necessidade de continuidade do estudo com vistas a uma ampliação numérica para se estabelecer outras características de comportamento relacionadas com os episódios de crises hipertensivas mesmo em vigência de um tratamento contínuo.

\section{REFERÊNCIAS}

(1) Kohlmann Jr O. Tratamento medicamentoso. In: Quartas diretrizes brasileiras de hipertensão: mesa redonda. Rio de Janeiro: Publicações Científicas; 2002. Cap. 6, p. 15-22.

(2) Damasceno CF, Moreira MJB, Lima RCFV, Araújo TL. Leito-dia: a experiência de atendimento nas urgências da diabetes e da hipertensão arterial. In: Livro resumo do $52^{\circ}$ Congresso Brasileiro de Enfermagem; 2000 out. 21-26; Recife. Recife: ABEnSeção PE; 2000. p. 12.

(3) Araújo GBS. Adesão ao tratamento antihipertensivo: análise conceitual. [dissertação]. João Pessoa (PB): Centro de Ciências da Saúde da UFPB; 2002.

(4) Amaral CFS. Emergências hipertensivas. In: Amodeo C, Lima EG, Vasquez EC. Hipertensão arterial. São Paulo: Sarvier; 1997. Cap. 32, p. 27783.

(5) Sanchez CG, Pierin AMG, Mion Jr D. Comparação dos perfis dos pacientes hipertensos atendidos em Pronto-Socorro e em tratamento ambulatorial. Rev Esc Enferm USP 2004; 38(1): 90-8.

(6) Triviños ANS. Introdução à pesquisa em ciências sociais. São Paulo: Atlas; 1993. p. 120.

(7) Polit DF, Hungler BP. Fundamentos da pesquisa em enfermagem. $3^{\mathrm{a}}$ ed. Porto Alegre: Artes Médicas; 1995. p. 108-40.

(8) Conselho Nacional de Saúde. Resolução no 196, de 10 de novembro de 1996. Diretrizes e normas regulamentadoras de pesquisas envolvendo seres humanos. Bioética 1996; 4(2 Supl):15-25.
(9) Resende MMC. Fatores que dificultam o controle da hipertensão arterial à luz da estrutura conceitual da teoria de King [dissertação]. Belo Horizonte: Escola de Enfermagem da UFMG; 1998. p. 116.

(10) Lopes MVO, Araújo TL. Aspectos epidemiológicos de mulheres com angina pectoris. In: Damas-ceno MMC, Araújo TL, Fernandes AEC. Transtornos vitais no fim do século XX: diabetes mellitus, distúrbios cardiovasculares, câncer, AIDS, tuberculose e hanseníase. Fortaleza: Fundação Cearense de Pesquisa e Cultura; 1999. Pt. 2, cap.7, p. 55-62.

(11) Dantas RAS, Colombo RCR, Aguillar OM. Perfil de mulheres com infarto agudo de miocárdio segundo o modelo de "campo de saúde". Rev LatAm Enferm 1999; 7(3):63-8.

(12) Castro LD, Car MR. O cotidiano da vida de hipertensos: mudanças, resistências e reações. Rev Esc Enferm USP 2000; 35(2):145-53.

(13) Teixeira ACA. Adesão ao tratamento farmacológico da hipertensão arterial e seus determinantes em pacientes de ambulatório [dissertação]. Fortaleza: Departamento de Saúde Comunitária da Faculdade de Medicina da UFCE; 1998. p. 113.

(14) Kaplan NM. Resistant hypertension: what to do after trying "the usual". Geriatrics 1995; 50(5): 24-38.

(15) Ribeiro JM. Acompanhamento do paciente hipertenso. In: Amodeo C, Lima EG, Vasquez EC. Hipertensão arterial. São Paulo: Sarvier; 1997. Cap. 15, p. 115-7.

(16) Povoa R, Galvão L. Crises hipertensivas [online] São Paulo: Casa de Cardiopatia Hipertensiva; 2002; Disponível em: http://www.unifesp.br/ $\mathrm{dmed} /$ cardio/ch/crises.htm. (10 nov. 2002)

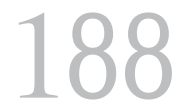

Rev Esc Enferm USP $2005 ; 39(2): 181-8$. 